one or two sentences to ensure the reader has grasped the salient point. Diagrams are illustrative and without too much detail. The use of analogies to relate everyday actions and experiences to those of flight are helpful.

An entire chapter is given over to turbulence and weather that for many anxious fliers causes great distress. Many of the scenarios that are expanded on are extremely rare. Even for the experienced flier it is helpful to know Captain Allright's diversion ratings, which are given out of 10, for such conditions as ice and thunderstorms through to a plane being hit by lightening. None scores more than 3/10.

For many, despite the theory, how to master their fear is what is more important Patricia Furness-Smith explains phobias and why they manifest. She likens the medical term phobia to an imp and uses this analogy to link an image that the reader can easily identify with. This use of analogy is developed further when she describes the lower brain, the limbic system, and the neocortex. The amygdala is likened to 'The General' and the autonomic nervous system.

Throughout the book there are short case histories further allowing the reader to identify with stressful situations that occur when having to take a flight. Panic attacks and the resulting emotional and physical symptoms are listed in detail to enable the reader to then use the "toolbox of techniques that are categorised using the letter 'R'. These are React, Regulate, Relax, and Rehearse.

The final sections of the book cover the 'how to'. There are detailed techniques that use cognitive behavioural therapy and breathing as the main methods of reducing stress and panic. Guided visualisation and positive thought are further techniques used to induce relaxation before the reader takes an imaginary trip that leaves home, encompasses check-in and boarding, the in-flight experience, and finally landing. This trip is interwoven with the exercises that previously the reader had practised.

This book is a useful adjuvant, that I, as a GP, could recommend to the phobic or fearful flier alongside the possible prescription for an anxiolytic. Explanations and practical techniques aim to give the anxious flier control over their destiny and to reclaim part of their lost life.

\section{Ian Kelso,}

E-mail: ian_kelsolayahoo.com

DOI: 10.3399/bjgp13X668320

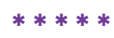

THE UNIVERSE WITHIN A SCIENTIFIC ADVENTURE

\section{NEIL SHUBIN}

Allen Lane, 2013

PB, 240pp, €20.00 978-1846142208

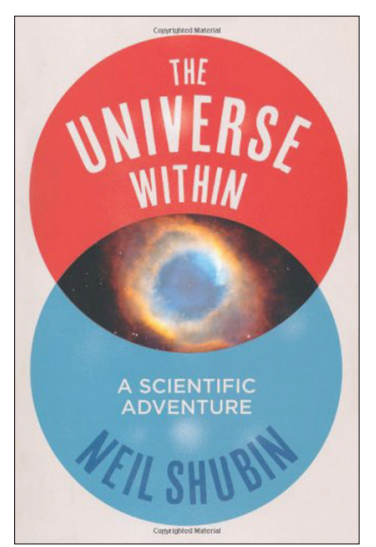

The Universe Within, by renowned palaeontologist Neil Shubin, is a short book about big topics. The goal of the book, as the dust jacket says, is to show how the one place where universe, solar system and planet merge is inside your body'. This is an enigmatic claim, and having read the book I'm still not entirely clear what it means.

This is not such a big issue, however, for Shubin takes us on a fascinating journey through the history of our universe, the formation of our solar system, the forces that created the earth and moon, and the evolutionary history of our planet - all in 200 easy-to-read pages! This is no small feat.

The book is arranged chronologically, and starts 13.7 billion years ago with the birth of the universe in the Big Bang. Shubin explains, in admirably clear terms, how the ball of energy that was the very early universe gave rise to simple elements like hydrogen, helium, and lithium.

Much later, heavier atoms such as carbon, oxygen, and nitrogen - so important in the biochemistry of Earth's organisms - were formed in fusion reactions deep in the heart of stars. When these element-forming stars exploded as supernovae, they spread out their atomic creations, which were eventually incorporated into the bodies of every creature on the planet. We are truly made of star dust.

Shubin has many more stories to tell than can be summarised in a short review.
He describes the formation of our solar system; how the 24-hour day/night cycle has led to the evolution of molecular body clocks in many species; how climatic changes 2 billion years ago set the stage for the evolution of multicellular animals; the role of continental drift in the history of our planet; and much, much more.

Throughout, Shubin's writing remains light and engaging, and the narrative is filled with thumbnail sketches of great and lesser-known scientists, as well as tales from Shubin's own paleontological adventures in the field. Although I felt that the 'universe within' conceit is a bit overstretched, this is a wonderfully broadranging, mind-expanding book. Read it, and you will better appreciate how intimately your existence is tied to the deep history of our universe.

\section{Dan Jones,}

\section{E-mail: dan.jonesवmultipledrafts.com}

DOI: 10.3399/bjgp13X668339 